\title{
Petrography and Metamorphism of the Metasedimentary Country-Rocks of the J acurici Valley Chromitite-Hosting Mafic-Ultramafic Complexes, Bahia, Northeastern Brazil.
}

\author{
Eliane A. Del Lama ${ }^{1}$, Maria A. F. Candia ${ }^{2}$, Gergely A. J. Szabó ${ }^{2}$ \\ ${ }^{1}$ FAPESP - pos-doctoral fellowship \\ ${ }^{2}$ Departamento de Mineralogia e Geotectônica - Instituto de G eociências - USP \\ Rua do Lago 562, CEP 05508-900, São Paulo, SP
}

Keywords: Jacurici Valley, metasediments, metamorphism, metasomatism.

\begin{abstract}
This paper deal with on the metasedimentary country-rocks of the chromite-bearing ultramafic rocks that occur in the "Jacurici River Valley Chromium District" northeastern Bahia, Brazil.

This region presents a complex geologic-petrologic framework of rocks that were intensely deformed, metamorphosed and transformed by metasomatic processes, making it difficult to interpret their evolutionary/metamorphic record.

Although the metasedimentary country rocks have also been affected by such processes, it is possible to distinguish evidence of a previous high-grade metamorphism that affected them.

Thermobarometric data for the observed mineralogical associations indicate P-T conditions around $750-800^{\circ} \mathrm{C}$ and $7-$ $8 \mathrm{~kb}$ for the metamorphic peak, based mainly on the presence of olivine in marbles and the cordierite-garnet-sillimanitespinel association in aluminous gneisses.
\end{abstract}

Palavras-chave: Vale do Rio Jacurici, metassedimentos, metamorfismo, metassomatismo.

\section{RESUMO}

Este trabalho enfoca as encaixantes metassedimentares das rochas ultramáficas, portadoras de mineralização de cromita, que ocorrem no nordeste do estado da Bahia.

Esta região, conhecida como "Distrito Cromitífero do Vale do Rio Jacurici", apresenta grande complexidade geológicapetrológica com rochas intensamente deformadas, metamorfizadas e transformadas por processos metassomáticos, o que dificulta a interpretação do registro metamórfico/evolutivo dessas rochas.

Apesar dos metassedimentos também terem sido afetados pelos processos acima, é possível recuperar registros do metamorfismo de alto grau.

Dados termobarométricos indicam condições P-T de $750-800^{\circ} \mathrm{Ce}$ 7-8 kb, baseados na presença de olivina nos mármores e na associação cordierita-granada-sillimanita-hercinita nos gnaisses aluminosos. 


\section{INTRODUCTION}

The study area is located in the Senhor do Bonfim $1 / 4$ degree sheet topographical map, Northeastern Bahia State, limited by the parallels $10^{\circ} 00^{\prime}$ and $11^{\circ} 00^{\prime} \mathrm{S}$ and by the meridians 39 $30^{\prime}$ ' and $39^{\circ} 50^{\prime} \mathrm{W}$ (Figure 1), and belongs to the Jacurici River Valley Chromian District.

This work focuses on the country-rocks of the chromitebearing ultramafic rocks that occur in the region. The country-rocks are represented by granulitic gneisses and other particular rock types such as marble, calc-silicate rocks, metaorthoclasite and aluminous gneiss. These particular rocks types will be detailed in this paper.

The area presents a complex geologic-petrologic framework with rocks intensely deformed, metamorphosed and transformed by metasomatic process, making it difficult to recover their evolutionary/metamorphic record.

Systematic geologic/petrologic investigations are scarce; only unpublished reports are available, made by request of the interested mining companies.

The aim of this paper is to describe the lithologic types not usually refered in published work, attempting to recover their original metamorphic imprint, as this kind of record is difficult to be recovered from the ultramafic rocks.

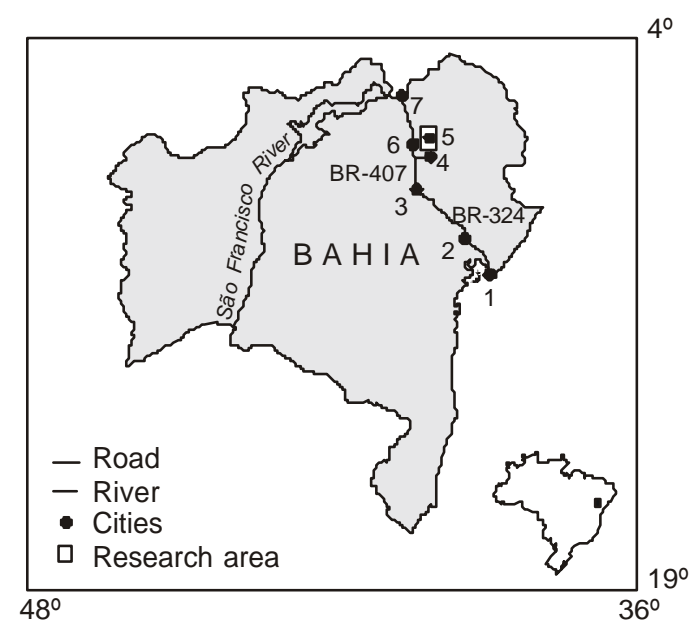

Figure 1: Localization and access roads of the researched area. (1- Salvador, 2- Feira de Santana, 3- Capim G rosso, 4- Itiúba, 5- Andorinha, 6- Senhor do Bonfim, 7- Juazeiro).

\section{GEOLOGICAL SETTING}

The chromite-bearing ultramafic rocks belong to the Caraíba Complex, outcropping along the Salvador-Curaçá Belt. The term Caraíba was used for the first time as a group by Barbosa $(1964,1970)$ for rocks that occur in the Curaçá river area. Figueiredo (1981) characterized this unit as a complex and studied its lithogeochemistry. Other denominations for this group are: Migmatitic-metamorphic Complex (CPRM, 1974 in Barbosa de Deus \& Viana, 1982) and Pedra Vermelha Complex (Barbosa de Deus \& Viana, 1982). The term Caraíba Complex is the designation adopted by Barbosa \& Dominguez (1996) in the 1:1.000.000 Geological Map of Bahia State (Figure 2).

The Caraíba Complex is constituted predominantly by hypersthene-bearing gneisses with tonalitic to granitic composition. These rocks present grey-greenish colour when fresh, and brown when altered, and exhibit a penetrative foliation striking N-S with steep dips. Migmatitic structures are common, with gabbro-dioritic paleosome.

Barbosa \& Dominguez (1996) interpret the Caraíba Complex rocks as belonging to the Transamazonic Cycle. In the Itabuna-Caraíba Complex, Silva et al. (1997) obtained U-Pb SHRIMP value of 2,695 Ma for the magmatic age and 2,072 Ma for the metamorphic age. Medrado mafic-ultramafic complex shows $\mathrm{U}-\mathrm{Pb}$ in zircon age of 2,059 Ma (Oliveira, 1998).

Mafic-ultramafic bodies generally occur interlayered with layers and lenses of carbonatic/calc-silicate rocks, quartz and aluminous gneiss, iron formation and graphitebearing schists. The sequence outcrops along a N-S direction parallel to the Itiúba Ridge located to the west (Figure 2), which is approximately $100 \mathrm{~km}$ long and $12 \mathrm{~km}$ wide. It has a syenitic composition and structural features indicating intrusive emplacement. The available $\mathrm{Rb}-\mathrm{Sr}$ isotopic data point to an age around $2.0 \mathrm{Ga}$ for this pluton (Conceição, 1990 in Barbosa \& Dominguez, 1996).

The chromite-bearing bodies are generally thick, specially when compared with other chromitites of important stratiform complexes, including Campo Formoso. Although they usually exhibit thicknesses from 5 to $8 \mathrm{~m}$ they may attain thicknesses up to $15 \mathrm{~m}$.

A possible stratigraphic sequence for the Jacurici Chromium District was proposed by Jardim de Sá (1984). From base to top he described:

-a Sequence of Supracrustal Rocks;

-Mafic-Ultramafic Bodies;

-G1 orthogneiss, with a predominantly acid composition, and displaying granulite facies parageneses. Several xenoliths of the supracrustal sequence are observed in this unit; 


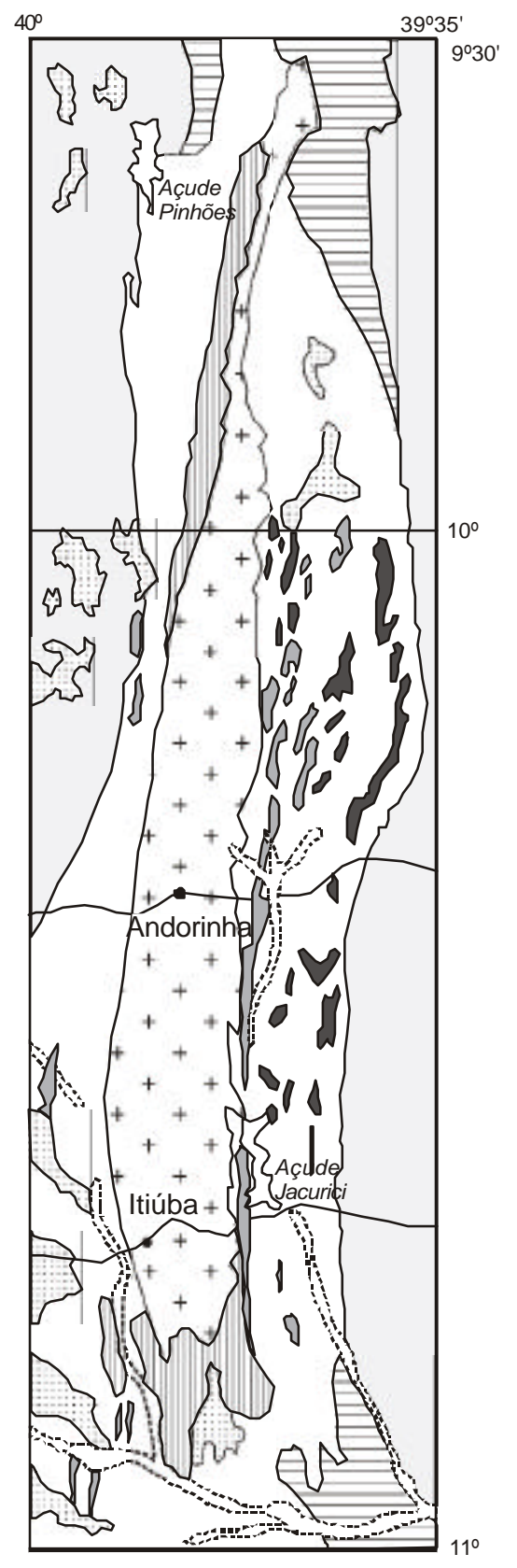

\section{QUATERNARY-HOLOCENE \\ Alluvium, sands and sandy clays}

TERTIARY-QUATERNARY

Terrigenous covers

\section{EARLY PROTEROZOIC}

Granites, monzonites and syenites

Syenites of Itiúba Ridge

\section{ARCHAEAN-EARLY PROTEROZOIC}

Caraíba Complex: granulitized tonalites, trondhjemites and granodiorites

Orthogneisses of amphibolite facies with subordinate supracrustal components and migmatites

Orthogneisses of amphibolite facies, sometimes migmatized

Basic granulites of the Salvador-Curaçá

Belt

Mafic-ultramafic sills of the Jacurici River Valley

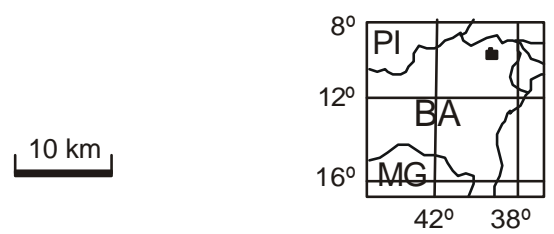

Figure 2: Geological map of the Jacurici River Valley area (Barbosa \& Dominguez, 1996).

-Mafic Dykes, amphibolitized, with decimetric to metric thicknesses;

-G2 granodiorite and tonalite, orthogneiss, with thin metamorphic layering and granulite to amphibolite facies parageneses;

-G3 granites, represented by the Itiúba Syenite and equivalent dykes.

\section{PETROGRAPHY}

The metasedimentary rocks of the Jacurici River Valley Chromium District are represented by spinel-sillimanite-cordierite-garnet gneiss, metaorthoclasite, marble, diopsidite and metasomatic rocks (e.g., phlogopitite, phlogopite gneiss and garnet epidosite).

The marble and the diopsidite are more frequent. The 
other lithologies are of restricted occurrence, being generally found in drill holes.

Chemical analyses of representative minerals are listed in Tables 1-3. The samples were analysed by electron microprobe at the Instituto de Geociências - USP. Analyses were performed on the JXA-8600 Superprobe (JEOL) with an accelerating voltage of $15 \mathrm{kV}$ and beam of $20 \mathrm{nA}$.

The mineral abbreviations are those of Kretz (1983).

\section{Spinel-Sillimanite-Cordierite-Garnet Gneiss}

This rock presents coarse granulometry with centimetric perthitic or mesoperthitic crystals. It is constituted by K-feldspar, quartz, cordierite, plagioclase, garnet, biotite, zircon and opaque minerals. Locally, sillimanite and green spinel also occur.

Plagioclase is albite $\left(\mathrm{An}_{6-10}\right)$ or oligoclase $\left(\mathrm{An}_{11-23}\right)-$ (Figure 3a).

Cordierite is distinguished by pinite alteration prod- ucts. Locally less altered grains are preserved with sillimanite and/or spinel inclusions. It can also involve garnet grains. Its composition is $\mathrm{Mg}$-rich $\left(\mathrm{X}_{\mathrm{Mg}}=0.8\right)-($ Table 1$)$.

Garnet is not very common. It shows poikiloblastic features and locally chloritized rims. Sillimanite and spinel inclusions occur, sillimanite being more common. It also appears as smaller crystals with subidioblastic to xenoblastic shapes. Garnet $\left(\mathrm{Alm}_{57-68} \operatorname{Prp}_{29-42}\right)$ may be zoned or not. Zoned crystals have core compositions enriched in $\mathrm{MgO}$ and depleted in $\mathrm{FeO}$ relative to the rim (Figure 4).

Biotite and phlogopite exhibt brown pleochroism and occur as irregular and small crystals. They are Tirich (Figure $5 \mathrm{~b}$ ) with variable $\mathrm{Fe}$ and $\mathrm{Mg}$ contents (Figure $5 \mathrm{~d})$.

Opaques are associated with biotite and phlogopite. Zircon appears as small rounded crystals and generally develops weak pleochroic haloes in cordierite.

Spinel shows strong green tonality. Its composition is that of a gahnitic hercynite $\left(\mathrm{X}_{\mathrm{Fe}}=0.43-0.64\right)$. $\mathrm{ZnO}$ content varies from 6 to $11 \%$ and $\mathrm{Fe}$ content decreases with increasing $\mathrm{Mg}$ content. Spinels included in garnet are $\mathrm{Mg}$ richer and Fe-poorer (Figure 6).

(a)
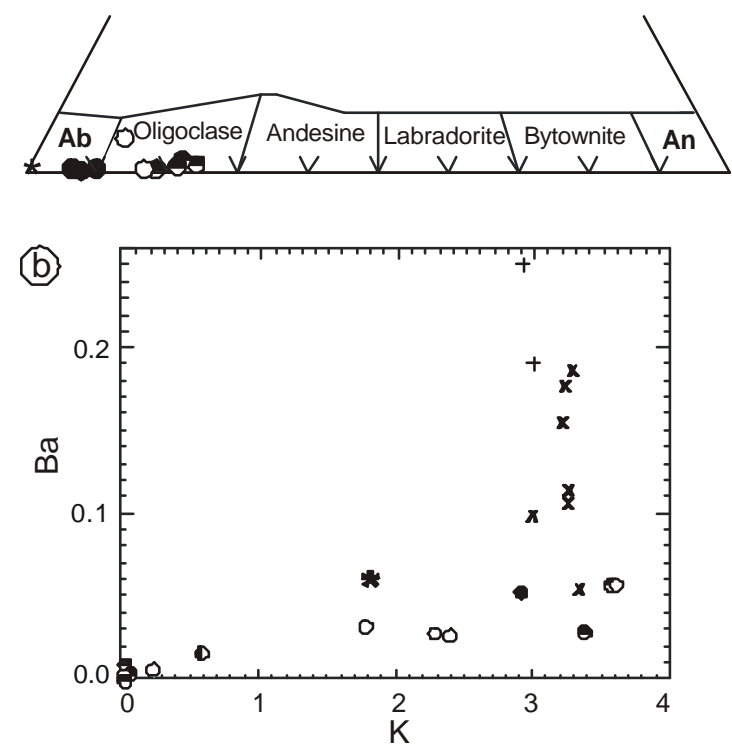

Aluminous gneiss

- Ma-132-1

- Ma-132-2

c Mas-127-2

- Mas-127-3

- Marbles (Ip-98-1, Med-4 4 Med-6)

$\bowtie$ Diopsidite (Med-98-32)

Metaorthoclasite

* Med-1

$x$ Med-7

+ Med-8

Metasomatic rocks

$\diamond$ Ri-01

- Vm-24

$\Delta \mathrm{Vm}-24$

V Vm-29

Figure 3: a- Compositional field for plagioclase from aluminous gneiss. $\mathrm{b}-\mathrm{K} \times \mathrm{Ba}$ diagram in p.f.u. (atoms per formula unit). 
Table 1: Representative analyses of feldspar, cordierite, garnet and spinel (samples Ma-132-1, Mas-127-2, Mas-127-3: aluminous gneiss; Med-1, Med-7, Med-8: metaorthoclasite; Vm-24: garnet epidosite).

\begin{tabular}{|c|c|c|c|c|c|c|c|c|c|c|c|c|}
\hline \multirow{3}{*}{$\begin{array}{l}\text { MINERAL } \\
\text { SAMPLE } \\
\text { ANALYSIS }\end{array}$} & \multicolumn{9}{|c|}{ feldspar } & & \multicolumn{2}{|c|}{ cordierite } \\
\hline & Mas-127-2 & Mas-127-2 & Mas-127-3 & Mas-127-3 & Ma-132-1 & Ma-132-1 & Med-1 & Med-7 & Med-8 & & Мa-132-1 & Ma-132-1 \\
\hline & 71 & 72 & 99 & 100 & 371 & 370 & 145 & 368 & 148 & & 376 & 388 \\
\hline $\mathrm{SiO}_{2}$ & 65.18 & 64.66 & 63.27 & 63.92 & 67.39 & 65.58 & 65.94 & 63.39 & 63.02 & & 49.11 & 49.44 \\
\hline $\mathrm{TiO}_{2}$ & 0.08 & 0.02 & 0.02 & 0.02 & 0.04 & 0.11 & 0.04 & 0.05 & 0.02 & & 0.04 & 0.01 \\
\hline $\mathrm{Al}_{2} \mathrm{O}_{3}$ & 22.31 & 18.49 & 23.14 & 18.42 & 21.06 & 18.92 & 18.97 & 18.94 & 19.36 & & 33.75 & 34.01 \\
\hline $\mathrm{FeO}$ & 0.02 & 0.05 & - & - & 0.05 & - & 0.38 & 0.03 & 0.06 & & 4.11 & 5.28 \\
\hline $\mathrm{MnO}$ & 0.00 & 0.01 & - & - & - & 0.01 & - & - & 0.02 & & - & - \\
\hline $\mathrm{MgO}$ & 0.02 & 0.00 & - & - & - & 0.00 & 0.02 & - & - & & 11.60 & 10.87 \\
\hline $\mathrm{BaO}$ & 0.04 & 0.79 & 0.10 & 0.77 & - & 0.72 & 0.83 & 2.53 & 3.44 & & n.d. & n.d. \\
\hline $\mathrm{SrO}$ & 0.06 & - & 0.03 & - & 0.03 & 0.02 & 0.12 & 0.07 & 0.18 & & n.d. & n.d. \\
\hline $\mathrm{ZnO}$ & n.d. & n.d. & n.d. & n.d. & n.d. & n.d. & n.d. & n.d. & n.d. & & 0.24 & 0.10 \\
\hline $\mathrm{CaO}$ & 3.41 & 0.08 & 4.39 & 0.01 & 1.65 & 0.25 & 0.11 & - & 0.02 & & - & - \\
\hline $\mathrm{Na}_{2} \mathrm{O}$ & 9.43 & 0.83 & 8.87 & 1.02 & 10.88 & 2.91 & 5.87 & 1.42 & 2.22 & & - & 0.03 \\
\hline $\mathrm{K} 2 \mathrm{O}$ & 0.13 & 15.32 & 0.19 & 15.01 & 0.08 & 12.60 & 7.84 & 13.81 & 12.21 & & - & - \\
\hline TOTAL & 100.69 & 100.24 & 100.01 & 99.18 & 101.16 & 101.13 & 100.11 & 100.22 & 100.54 & & 98.85 & 99.75 \\
\hline $\mathrm{AB}$ & 82.70 & 7.60 & 77.70 & 9.40 & 91.90 & 25.70 & 52.90 & 13.50 & 21.60 & $X_{M g}$ & 0.83 & 0.78 \\
\hline $\mathrm{AN}$ & 16.50 & 0.40 & 21.20 & 0.10 & 7.70 & 1.20 & 0.50 & 0.00 & 0.10 & & & \\
\hline OR & 0.70 & 92.10 & 1.10 & 90.50 & 0.40 & 73.10 & 46.50 & 86.50 & 78.30 & & & \\
\hline
\end{tabular}

\begin{tabular}{|c|c|c|c|c|c|c|c|c|c|c|c|}
\hline \multirow{3}{*}{$\begin{array}{l}\text { MINERAL } \\
\text { SAMPLE } \\
\text { ANALYSIS }\end{array}$} & \multicolumn{7}{|c|}{ garnet } & \multicolumn{4}{|c|}{ s p in e 1} \\
\hline & Ma-132-1 & Ma-132-1 & Mas-127-2 & Mas-127-2 & Mas-127-2 & Vm-24 & & Mas-127-2 & Mas-127-3 & Ma-132-1 & Ma-132-1 \\
\hline & 333 & 387 & 51 & 52 & 55 & 292 & & 48 & 69 & 374 & 379 \\
\hline $\mathrm{SiO}_{2}$ & 38.43 & 37.95 & 38.20 & 38.86 & 38.02 & 37.25 & & 0.02 & 0.01 & - & - \\
\hline $\mathrm{TiO}_{2}$ & 0.03 & - & - & - & - & 0.67 & & 0.02 & 0.03 & 0.04 & - \\
\hline $\mathrm{Al}_{2} \mathrm{O}_{3}$ & 22.87 & 21.76 & 21.98 & 22.24 & 21.86 & 17.15 & & 58.85 & 58.53 & 57.63 & 61.14 \\
\hline $\mathrm{Cr}_{2} \mathrm{O}_{3}$ & - & - & - & - & - & 2.80 & & - & 0.03 & 0.28 & 0.04 \\
\hline $\mathrm{Fe}_{2} \mathrm{O}_{3}$ & 1.58 & 1.80 & 1.69 & 1.55 & 1.76 & 6.80 & & 0.05 & 1.70 & 2.93 & 1.33 \\
\hline $\mathrm{FeO}$ & 27.08 & 30.75 & 28.92 & 26.57 & 30.06 & 6.12 & & 19.02 & 24.94 & 26.76 & 19.03 \\
\hline $\mathrm{Y}_{2} \mathrm{O}_{3}$ & - & 0.06 & 0.01 & 0.02 & - & 0.04 & & n.d. & n.d. & n.d. & n.d. \\
\hline $\mathrm{MnO}$ & 0.11 & 0.17 & 0.51 & 0.47 & 0.71 & 0.58 & & 0.02 & 0.08 & - & - \\
\hline $\mathrm{MgO}$ & 11.03 & 7.89 & 8.16 & 10.20 & 7.29 & 0.88 & & 6.96 & 5.91 & 5.83 & 10.60 \\
\hline $\mathrm{NiO}$ & n.d. & n.d. & n.d. & n.d. & n.d. & n.d. & & - & 0.03 & 0.04 & - \\
\hline $\mathrm{ZnO}$ & n.d. & n.d. & n.d. & n.d. & n.d. & n.d. & & 11.18 & 7.35 & 5.59 & 6.58 \\
\hline $\mathrm{CaO}$ & 0.35 & 0.38 & 0.41 & 0.53 & 0.56 & 26.47 & & - & - & - & - \\
\hline $\mathrm{Na}_{2} \mathrm{O}$ & - & 0.01 & 0.02 & 0.01 & 0.03 & - & & n.d. & n.d. & n.d. & n.d. \\
\hline $\mathrm{K}_{2} \mathrm{O}$ & 0.01 & 0.01 & - & - & 0.01 & - & & n.d. & n.d. & n.d. & n.d. \\
\hline $\mathrm{Nb}_{2} \mathrm{O}_{5}$ & n.d. & n.d. & n.d. & n.d. & n.d. & n.d. & & - & - & - & - \\
\hline $\mathrm{P}_{2} \mathrm{O}_{5}$ & n.d. & n.d. & n.d. & n.d. & n.d. & n.d. & & - & - & - & - \\
\hline TOTAL & 101.34 & 100.59 & 99.73 & 100.31 & 100.11 & 98.09 & & 96.12 & 98.59 & 99.11 & 98.73 \\
\hline $\mathrm{X}_{\mathrm{Mg}}$ & 0.42 & 0.31 & 0.33 & 0.40 & 0.29 & 0.04 & $\mathrm{XMg}_{\mathrm{Mg}}$ & 0.30 & 0.25 & 0.25 & 0.43 \\
\hline ALM & 48.51 & 59.02 & 64.98 & 57.86 & 67.58 & 14.51 & $\mathrm{X}_{\mathrm{Fe}}$ & 0.46 & 0.59 & 0.64 & 0.43 \\
\hline PRP & 50.07 & 39.14 & 32.68 & 39.61 & 29.20 & 3.73 & $\mathrm{Xzn}$ & 0.24 & 0.15 & 0.12 & 0.13 \\
\hline AND & 1.12 & 1.37 & 1.18 & 1.48 & 1.61 & 21.76 & & & & & \\
\hline GRS & - & - & - & - & - & 49.20 & & & & & \\
\hline
\end{tabular}

\section{Quartite}

It is only locally observed. Small tabulate orientated crystals of green mica are commonly observed, with green pale pleochroism. Its composition is that of chromian muscovite ("fuchsite") with variable $\mathrm{Cr}_{2} \mathrm{O}_{3}$ contents (1.5$4.5 \%$, meanly around $4 \%$, Table 2 ).

\section{Metaorthoclasite}

These rocks are formed predominantly by alkaline feldspar, with subordinate clinopyroxene and amphibole. Mesoperthites are common. The name orthoclasite was adopted based exclusively on the mineral association of this rock, with no genetic implication whatsoever. The protholith of this rock is currently under study. 

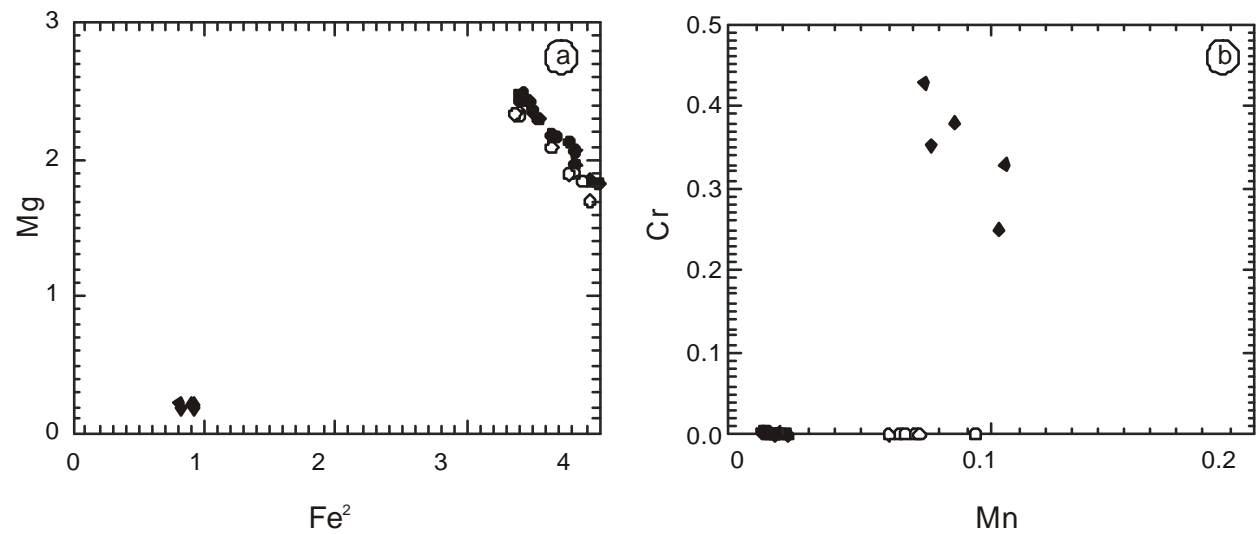

Figure 4: a- $\mathrm{Fe}^{+2} \times \mathrm{Mg}$ diagram for garnet in aluminous gneiss and garnet epidosite. b- $\mathrm{Mn} \times \mathrm{Cr}$ diagram for garnet in aluminous gneiss and garnet epidosite. Values in p.f.u. Symbols as in Figure 3.
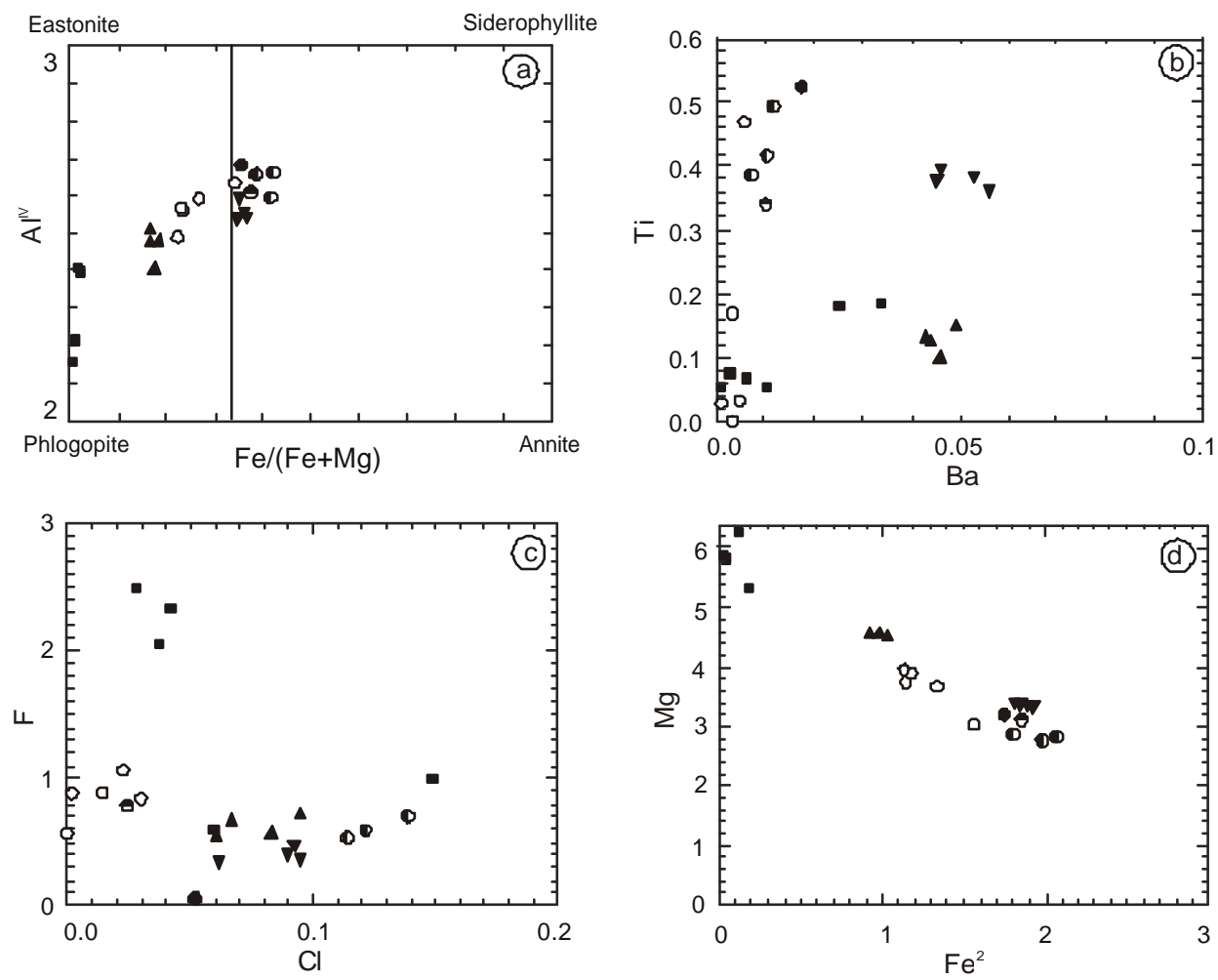

Figure 5: a- Compositional field for biotite. b- Ba xTi diagram. c- $\mathrm{Cl}$ x F diagram. d$\mathrm{Fe}^{+2} \times \mathrm{Mg}$ diagram. Values in p.f.u. Symbols as in Figure 3. 

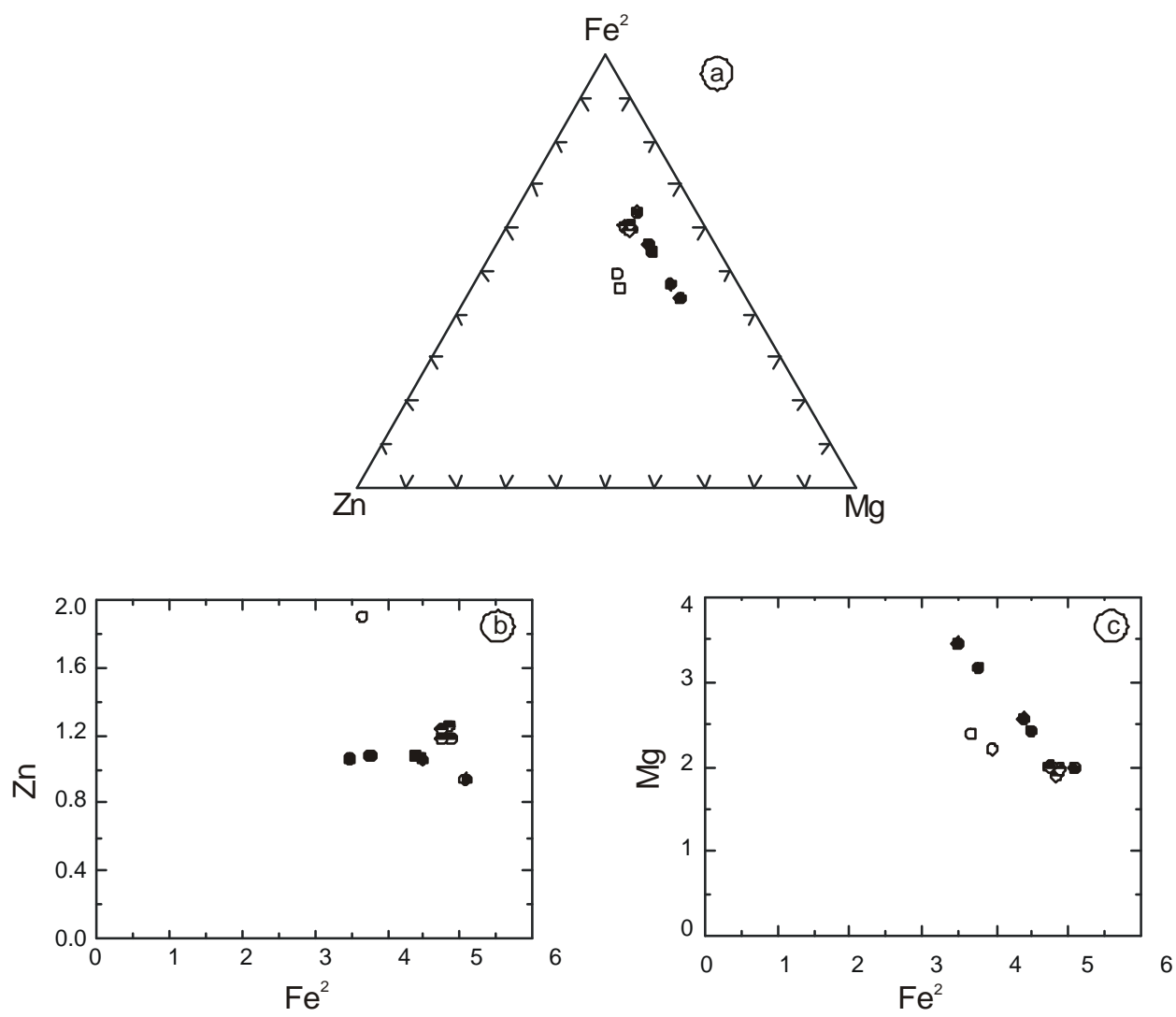

Figure 6: a- $\mathrm{Fe}^{+2} \times \mathrm{Zn} \times \mathrm{Mg}$ diagram for spinel from aluminous gneiss. b- $\mathrm{Fe}^{+2} \times \mathrm{Zn}$ diagram. C- $\mathrm{Fe}^{+2} \times \mathrm{Mg}$ diagram. Values in p.f.u. Symbols as in Figure 3.

The K-feldspar has high $\mathrm{BaO}$ content (Figure $3 \mathrm{~b}$ ), with concentrations ranging from 0.8 to $3.4 \%$ (Table 1 ).

Clinopyroxenes are diopside (sample Med-8), salite (Med-7) and Fe-salite (Med-1) (Figure 7).

Amphibole is actinolite and seems to be originated from clinopyroxene substitution. It shows greenish colour and is also very altered.

Apatite and sphene occur as well developed crystals. Apatite is common and can reach up to $4 \mathrm{~mm}$.

\section{Marble}

The serpentine marble is a very characteristic lithology of the metasedimentary sequence. Its particularity are the serpentine "balls" present in the carbonates. Its mineralogy is constituted predominantly by granoblastic carbonates, with olivine "balls" partially or completely substituted by serpentine. Phlogopite, chlorite, talc, apatite and opaques are minor constituents. Olivine is locally preserved. It has a $\mathrm{Fo}_{96-99}$ composition, being $\mathrm{Mg}$-richer than the olivines of the ultramafic rocks. Its low $\mathrm{NiO}$ content $(0.04 \%)$ indicates a different genesis with regard to the olivine of the ultramafic sequence. Serpentine occurs in the "balls" and along threads or bands.

Based on textural features, antigorite seems to prevail among serpentines. Chrysotile, that is more fibrous, also occurs, interlocked with and involving antigorite. In some samples, there are disseminated interstitial carbonates. $\mathrm{FeO}, \mathrm{MgO}$ and $\mathrm{Al}_{2} \mathrm{O}_{3}$ contents in serpentines present small variations. Phlogopite seems to be late and is deformed. In the marble, phlogopite practically does not have $\mathrm{Cr}_{2} \mathrm{O}_{3}$, and presents $\mathrm{MgO}, \mathrm{TiO}_{2}, \mathrm{BaO}$ and $\mathrm{F}$ variations. Its $\mathrm{F}$ content is higher than in other petrographic types (Figure 5c).

Brownish balls of fibrous aggregates also occur in the marble and seems to have been phlogopite that altered to chlorite. Chlorite has anomalous blue birefringence, with penninite composition. Apatite is colourless and occurs as 
Table 2: Representative analyses of mica, clinopyroxene, olivine and amphibole (samples Ma-132-1, Ma-132-2, Mas-1272, Mas-127-2, Mas-127-3: aluminous gneiss; V-98: quartite; Med-1, Med-7, Med-8: metaorthoclasite; Ip-98-1, Med-4 ${ }_{B}$ Med-6: marble; Med-98-32: diopsidite; Ri-01, Vm-24, Vm-24, $\mathrm{Vm}-29$ : metasomatic rocks).

\begin{tabular}{|c|c|c|c|c|c|c|c|c|c|c|c|c|c|}
\hline $\begin{array}{l}\text { MINERAL } \\
\text { SAMPLE }\end{array}$ & Mas-127-2 & Mas-127-2 & Mas-127-3 & 3 Ма-132-1 & Мa-132-2 & $\begin{array}{l}\mathrm{m} \mathrm{ic} \mathrm{a} \\
\mathrm{Vm}-24_{\mathrm{A}}\end{array}$ & $\mathrm{Vm}-29$ & Ip-98-1 & Med-4B & Med-6 & Ri-01 & V-98 & $\mathrm{Vm}-24$ \\
\hline ANALYSIS & 42 & 45 & 95 & 382 & 38 & 1 & 5 & 49 & 112 & 122 & 8 & 4 & 295 \\
\hline $\mathrm{SiO}_{2}$ & 38.22 & 37.05 & 36.01 & 35.97 & 36.20 & 37.91 & 36.68 & 41.55 & 39.94 & 39.14 & 45.88 & 45.14 & 45.36 \\
\hline $\mathrm{TiO}_{2}$ & 0.28 & 4.32 & 3.05 & 4.71 & 3.76 & 1.19 & 3.24 & 0.63 & 1.75 & 0.62 & - & 0.24 & - \\
\hline $\mathrm{Al}_{2} \mathrm{O}_{3}$ & 20.33 & 18.75 & 17.40 & 17.03 & 17.74 & 16.40 & 15.72 & 14.06 & 16.00 & 14.46 & 40.86 & 35.45 & 37.17 \\
\hline $\mathrm{Cr}_{2} \mathrm{O}_{3}$ & - & - & 0.01 & 0.01 & 0.05 & 0.18 & 0.08 & 0.04 & 0.03 & 0.02 & - & 4.52 & - \\
\hline $\mathrm{FeO}$ & 9.91 & 12.91 & 14.79 & 14.20 & 16.79 & 7.60 & 14.58 & 0.36 & 1.44 & 1.21 & 0.12 & 0.86 & 0.16 \\
\hline $\mathrm{MnO}$ & - & 0.02 & 0.02 & - & 0.01 & 0.04 & 0.08 & 0.02 & - & 0.01 & - & 0.16 & 0.02 \\
\hline $\mathrm{MgO}$ & 18.40 & 14.02 & 13.94 & 14.58 & 12.88 & 21.16 & 15.30 & 28.11 & 25.28 & 30.13 & 0.41 & 0.41 & - \\
\hline $\mathrm{BaO}$ & 0.01 & 0.11 & 0.16 & 0.31 & 0.17 & 0.76 & 0.97 & 0.11 & 0.47 & 0.11 & 0.09 & 0.03 & - \\
\hline $\mathrm{CaO}$ & - & - & - & - & - & 0.02 & 0.02 & 0.03 & 0.14 & 0.08 & 0.27 & - & 0.13 \\
\hline $\mathrm{Na}_{2} \mathrm{O}$ & 0.08 & 0.07 & 0.06 & 0.09 & 0.16 & 0.17 & 0.12 & 0.25 & 0.55 & 0.32 & 0.18 & 0.32 & 0.09 \\
\hline $\mathrm{K}_{2} \mathrm{O}$ & 9.63 & 9.85 & 9.9 & 9.18 & 9.80 & 9.17 & 9.36 & 10.28 & 9.23 & 6.88 & 10.49 & 10.08 & 10.78 \\
\hline $\mathrm{F}$ & 1.07 & 0.57 & 0.78 & 0.06 & 0.54 & 0.67 & 0.47 & 2.04 & 0.59 & 0.97 & - & 0.63 & - \\
\hline $\mathrm{Cl}$ & 0.02 & - & 0.02 & 0.05 & 0.11 & 0.07 & 0.09 & 0.04 & 0.06 & 0.15 & 0.01 & 0.01 & 0.02 \\
\hline $\mathrm{H} 2 \mathrm{O}$ & 3.71 & 3.87 & 3.6 & 4.02 & 3.78 & 3.78 & 3.78 & 3.33 & 3.97 & 3.74 & 4.68 & 4.25 & 4.46 \\
\hline TOTAL & 101.66 & 101.54 & 99.77 & 100.21 & 101.98 & 99.11 & 100.49 & 100.83 & 99.44 & 97.82 & 102.98 & 102.09 & 98.18 \\
\hline $\mathrm{X}_{\mathrm{Mg}}$ & 0.77 & 0.66 & 0.63 & 0.65 & 0.58 & 0.83 & 0.65 & 0.99 & 0.97 & 0.98 & 0.86 & 0.46 & 0.00 \\
\hline MINERAL & \multicolumn{6}{|c|}{ clinopyroxene } & \multicolumn{4}{|c|}{ olivine } & \multicolumn{3}{|c|}{ amphibole } \\
\hline SAMPLE & Med-1 & Med-7 & Med-8 N & Med-98-32 & \multicolumn{2}{|r|}{$\mathrm{Vm}-24 \mathrm{~A}$} & \multicolumn{4}{|c|}{$-98-1 \quad$ Med-4в } & \multicolumn{2}{|c|}{ Med-7 } & Ri-01 \\
\hline ANALYSIS & 137 & 395 & 138 & 4 & 10 & 20 & \multicolumn{4}{|c|}{128} & \multicolumn{2}{|r|}{393} & 11 \\
\hline $\mathrm{SiO}_{2}$ & 50.64 & 53.15 & 53.12 & 55.11 & 52.32 & 53.17 & \multicolumn{4}{|c|}{41.91} & \multicolumn{2}{|r|}{55.86} & 55.72 \\
\hline $\mathrm{TiO}_{2}$ & 0.08 & - & 0.24 & - & 0.27 & - & \multicolumn{4}{|c|}{0.00} & \multicolumn{2}{|r|}{-} & 0.29 \\
\hline $\mathrm{Al}_{2} \mathrm{O}_{3}$ & 0.73 & 0.87 & 1.95 & 0.29 & 3.68 & 1.02 & \multicolumn{4}{|c|}{-} & \multicolumn{2}{|r|}{0.42} & 2.21 \\
\hline $\mathrm{Cr}_{2} \mathrm{O}_{3}$ & - & 0.04 & 0.03 & - & - & 0.10 & \multicolumn{4}{|c|}{-} & \multicolumn{2}{|r|}{0.02} & 0.08 \\
\hline $\mathrm{FeO}$ & 18.89 & 6.89 & 4.02 & 0.20 & 3.40 & 5.19 & \multicolumn{4}{|c|}{4.00} & \multicolumn{2}{|r|}{8.12} & 4.44 \\
\hline $\mathrm{MnO}$ & 0.27 & 0.63 & 0.64 & 0.07 & 0.16 & 0.17 & \multicolumn{4}{|c|}{0.34} & \multicolumn{2}{|r|}{0.47} & 0.09 \\
\hline $\mathrm{NiO}$ & 0.01 & - & 0.03 & - & - & - & \multicolumn{4}{|c|}{0.03} & \multicolumn{2}{|r|}{ n.d. } & n.d. \\
\hline $\mathrm{MgO}$ & 6.39 & 13.86 & 14.15 & 19.04 & 15.70 & 16.21 & \multicolumn{4}{|c|}{52.68} & & 19.09 & 20.62 \\
\hline $\mathrm{CaO}$ & 19.84 & 24.07 & 24.24 & 25.33 & 24.90 & 23.29 & & 0.03 & 0.03 & & & 12.83 & 13.79 \\
\hline $\mathrm{Na}_{2} \mathrm{O}$ & 2.09 & 0.42 & 0.63 & 0.08 & 0.11 & 0.30 & & - & - & & & 0.12 & 0.16 \\
\hline $\mathrm{BaO}$ & n.d. & n.d. & n.d. & n.d. & n.d. & n.d. & & n.d. & n.d. & & & - & - \\
\hline $\mathrm{K}_{2} \mathrm{O}$ & n.d. & n.d. & n.d. & n.d. & n.d. & n.d. & & n.d. & n.d. & & & 0.03 & 0.02 \\
\hline $\mathrm{F}$ & n.d. & n.d. & n.d. & n.d. & n.d. & n.d. & & n.d. & n.d. & & & - & - \\
\hline $\mathrm{Cl}$ & n.d. & n.d. & n.d. & n.d. & n.d. & n.d. & & n.d. & n.d. & & & - & - \\
\hline TOTAL & 98.93 & 99.92 & 99.05 & 100.13 & 100.53 & 99.44 & & 102.4 & 98.98 & & & 96.94 & 97.34 \\
\hline WO & 45.40 & 48.89 & 51.00 & 48.68 & 50.27 & 46.56 & & & & & & & \\
\hline EN & 20.40 & 39.17 & 41.40 & 50.91 & 44.11 & 45.09 & & & & & & & \\
\hline $\mathrm{FS}$ & 34.20 & 11.94 & 7.60 & 0.41 & 5.62 & 8.36 & & & & & & & \\
\hline
\end{tabular}

relatively well developed crystals, up to $3 \mathrm{~cm}$ (it is usually millimetric). Opaques, mainly magnetite, occur as dispersed subidioblastic crystals. They may outline the chlorite balls.

The carbonates can occur with a serpentine aureole, suggesting a reaction rim. They seem to assimilate the serpentine, substituting it. Sometimes they have indented contacts. Carbonates are interstitial to or cut across serpentine, indicating a carbonatation process after serpentinization.

\section{Diopsidite}

These rocks are characterized by well developed aggregates of diopside (Figure 7). The crystals are altered and very fractured. They present irregular, rounded and prismatic shapes, and may exhibit polygonal texture. Diopside grain size is variable ranging from finely recrystallized portions up to $7 \mathrm{~mm}$ crystals. Fine phyllosilicates are in the diopside grains contacts. Discontinuous serpentine veins, and chlorite and carbonate veins, cut randomly the clinopyroxenite. 

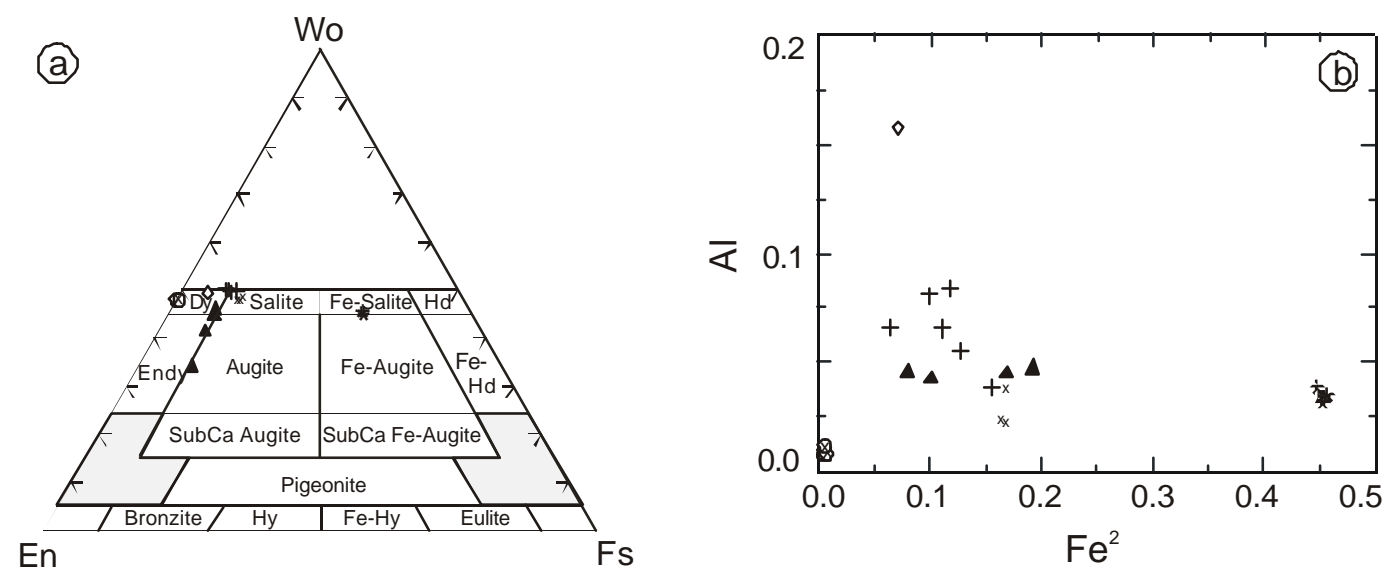

Figure 7: a- Compositional field for clinopyroxene. b- $\mathrm{Fe}^{+2} \times \mathrm{Al}$ diagram. Values in p.f.u. Symbols as in Figure 3.

\section{Rocks with phlogopite and/or clinozoisite}

The formation of phlogopite and/or biotite seems to be linked to a late metasomatic process that affected all rocks, both metasedimentary and ultramafic, with varying intensity, on behalf of the preferential fluid percolation paths. The effect of this process upon the metasedimentary rocks is noticed by frequent phlogopite and/or biotite development, concentrated in bands and veins, or randomly distributed in the rock. Phlogopite and biotite are Ba-rich. The metasomatic process is also observed in the ultramafic sequence, where the rocks are cut or involved by phlogopite.

\section{Hydrothermalized clinopyroxenite}

These rocks are composed predominantly by clinozoisite and phlogopite or biotite, with clinopyroxene remmants indicating hydrothermalized diopsidites. They present a banded aspect. Clinozoisite has brownish colour and occurs as prismatic crystals that seem to replace clinopyroxene; a crystal with clinopyroxene core and clinozoisite rims has been observed. Some clinozoisite crystals are zoned with epidote cores. Phlogopite (sample $\left.\mathrm{Vm}-24_{\mathrm{A}}\right)$ and biotite $(\mathrm{Vm}-29)$ occur as tabulate, isolated or aggregated crystals, with random disposition. It is a Barich variety (Figure 5b) and may show brown or brownish green colours, due to chloritization. Clinopyroxene is mainly diopside (samples Ri-01 and $\mathrm{Vm}-24_{\mathrm{A}}$ ) and endiopside and augite $\left(\mathrm{Vm}-24_{\mathrm{A}}\right)$ - Figure $7 \mathrm{a}$. It occurs as irregular, dirty and altered crystals, and may be impregnated by opaques. Amphibole is actinolite and seems to substitute clinopyroxene. Serpentine occurs interstitially. Carbonates occur associated with clinozoisite. Apatite may be common, as round or subidioblastic crystals. Sphene appears as small round crystals. Plagioclase, when present, is completely sericitized and occurs in the clinozoisiteand sphene-rich bands. Carbonate veins are also observed.

\section{G arnet epidosite}

Epidote/clinozoisite of brownish colour appears as prismatic crystals or in aggregates. It presents localized yellowish, heterogeneous pleochroism. Clinozoisite is seemingly replaced by epidote, as shown by its anomalous blue birefringence changing to yellow-blue birefringence. Fe and $\mathrm{Al}$ contents are variable (Table 3). Garnet shows irregular shapes, with fragments of larger crystals with yellowish colours, intimately associated with epidote. It is Ca-rich with $\mathrm{X}_{\mathrm{Ca}}=0.8 . \mathrm{Cr}_{2} \mathrm{O}_{3}$ content is high $(2-3 \%$ Figure $4 \mathrm{~b}$ ), and $\mathrm{Ti}$ and $\mathrm{Fe}^{+3}$ also accur. Completely sericitized plagioclase occurs interstitially to epidote. Opaques appear as xenoblastic crystals, may be well developed or not, and are relatively common. Sphene of brown colour occurs as minuscule punctuations all over the whole rock. Apatite is also present, and has higher $\mathrm{SrO}$ content than in other samples (Table 3). Locally muscovite is observed (Table 2). Carbonates occur impregnating the assemblage, associated with alteration. 
Table 3: Representative analyses of apatite, serpentine and epidote (samples Ip-98-1, Med-4 ${ }_{B}$, Med-6: marble; Med-7: metaorthoclasite; Ri-01, Vm-24, Vm-24, Vm-29: metasomatic rocks).

\begin{tabular}{|c|c|c|c|c|c|c|c|c|c|c|c|c|c|}
\hline MINERAL & \multicolumn{5}{|c|}{ apatite } & \multicolumn{4}{|c|}{ serpentine } & \multicolumn{4}{|c|}{ epidote } \\
\hline SAMPLE & Ip-98-1 & Med-7 & Vm-24 & $\mathrm{Vm}-24_{\mathrm{A}}$ & Vm-29 & Ip-98-1 & Med- $4_{\text {в }}$ & Med-4 & Med-6 & Ri-01 & $\mathrm{Vm}-24$ & Vm-24 & Vm-24 \\
\hline ANALYSIS & 55 & 399 & 308 & 24 & 28 & 51 & 111 & 114 & 121 & 14 & 325 & 326 & 328 \\
\hline $\mathrm{SiO}_{2}$ & 0.06 & 0.48 & 0.06 & 0.34 & 0.45 & 39.66 & 39.49 & 42.07 & 41.31 & 39.21 & 37.46 & 39.09 & 38.27 \\
\hline $\mathrm{TiO}_{2}$ & n.d. & n.d. & n.d. & n.d. & n.d. & - & 0.02 & 0.01 & 0.04 & - & 0.01 & 0.03 & - \\
\hline $\mathrm{Al}_{2} \mathrm{O}_{3}$ & n.d. & n.d. & n.d. & n.d. & n.d. & 0.09 & 0.28 & 0.12 & 0.83 & 35.65 & 26.62 & 33.28 & 29.90 \\
\hline $\mathrm{Cr}_{2} \mathrm{O}_{3}$ & n.d. & n.d. & n.d. & n.d. & n.d. & - & - & 0.01 & - & - & - & - & - \\
\hline $\mathrm{FeO}$ & - & & 0.02 & 0.09 & 0.22 & 0.45 & 3.83 & 1.54 & 1.06 & 0.46 & 8.18 & 1.94 & 5.40 \\
\hline $\mathrm{MnO}$ & 0.01 & 0.02 & 0.02 & - & 0.02 & 0.20 & 0.07 & 0.03 & 0.06 & 0.06 & 0.00 & 0.02 & 0.02 \\
\hline $\mathrm{MgO}$ & - & 0.01 & 0.01 & 0.05 & - & 41.34 & 40.18 & 41.55 & 40.53 & 0.29 & 0.01 & 0.02 & 0.01 \\
\hline $\mathrm{CaO}$ & 55.18 & 53.40 & 54.73 & 53.17 & 54.39 & 0.02 & 0.04 & 0.03 & 0.05 & 24.34 & 23.42 & 24.04 & 24.01 \\
\hline $\mathrm{BaO}$ & 0.05 & 0.11 & 0.12 & 0.00 & 0.00 & 0.04 & 0.01 & 0.12 & 0.08 & 0.07 & - & - & - \\
\hline $\mathrm{SrO}$ & 0.01 & 0.07 & - & 1.15 & 0.44 & n.d. & n.d. & n.d. & n.d. & 0.27 & - & - & - \\
\hline $\mathrm{Na}_{2} \mathrm{O}$ & 0.04 & 0.08 & - & 0.07 & 0.04 & - & 0.01 & 0.01 & 0.02 & - & - & - & - \\
\hline $\mathrm{K}_{2} \mathrm{O}$ & n.d. & n.d. & n.d. & n.d. & n.d. & 0.01 & 0.01 & - & 0.01 & - & - & 0.01 & - \\
\hline $\mathrm{P}_{2} \mathrm{O}_{5}$ & 41.21 & 40.47 & 41.75 & 41.72 & 42.13 & n.d. & n.d. & n.d. & n.d. & n.d. & n.d. & n.d. & n.d. \\
\hline $\mathrm{La}_{2} \mathrm{O}_{3}$ & - & 0.38 & 0.01 & 0.18 & 0.09 & n.d. & n.d. & n.d. & n.d. & - & - & - & 0.09 \\
\hline $\mathrm{Ce}_{2} \mathrm{O}_{3}$ & - & 0.50 & - & 0.43 & 0.73 & n.d. & n.d. & n.d. & n.d. & - & 0.02 & - & 0.06 \\
\hline $\mathrm{Y}_{2} \mathrm{O}_{3}$ & n.d. & n.d. & n.d. & n.d. & n.d. & n.d. & n.d. & n.d. & n.d. & 0.04 & 0.01 & - & 0.02 \\
\hline F & 4.07 & 2.84 & 2.92 & 3.57 & 3.67 & 0.03 & 0.02 & 0.04 & 0.07 & n.d. & n.d. & n.d. & n.d. \\
\hline $\mathrm{Cl}$ & 0.32 & 0.69 & 0.33 & 0.53 & 0.25 & 0.07 & 0.42 & 0.04 & 0.08 & n.d. & n.d. & n.d. & n.d. \\
\hline TOTAL & 100.95 & 99.03 & 99.97 & 101.31 & 102.42 & 81.90 & 84.26 & 85.55 & 84.09 & 100.38 & 95.72 & 98.43 & 97.79 \\
\hline
\end{tabular}

\section{METAMORPHISM}

Metamorphic aspects were detailed for metasedimentary rock types, which are more favourable for recovering the record of the metamorphic processes which took place in the area.

The marbles present a very simple mineralogy, essentially represented by olivine and calcite, with olivine replaced by serpentine.

As observed in Figure 8, the forsterite-calcite \pm dolomite association is stable under conditions above reactions 5 and 6. Carbonate from the analysed samples corresponds to calcite, however the presence of dolomite can not be excluded. Diopside and tremolite are not observed, if these phases existed, they were already transformed.

Considering that the metamorphism is essentially anhydrous, developed under high $\mathrm{XCO}_{2}$ conditions, the forsteritecalcite association has probably derived from the reaction $\mathrm{Di}+3 \mathrm{Dol}=4 \mathrm{Cal}+2 \mathrm{Fo}+2 \mathrm{CO}_{2}$. The variation of the equilibrium conditions as a function of pressure for different values of $\mathrm{T}-\mathrm{XCO}_{2}$ are presented in Figure 9, obtained from Berman (1991). In the 4 to $7 \mathrm{~kb}$ pressure range, the association is stable for temperatures up to $710-810^{\circ} \mathrm{C}$, respectively.

Olivine serpentinization possibly occured under distinct conditions: as seen in Figure 10, serpentine has its stability limited to a field below approximately $500^{\circ} \mathrm{C}$ and of low $\mathrm{XCO}_{2}$ (around 5\%). If $\mathrm{XCO}_{2}$ is higher, the Tlc $+\mathrm{Mgs}$ or Qtz + Mgs (see, respectively, curves 3 and 5 of Figure

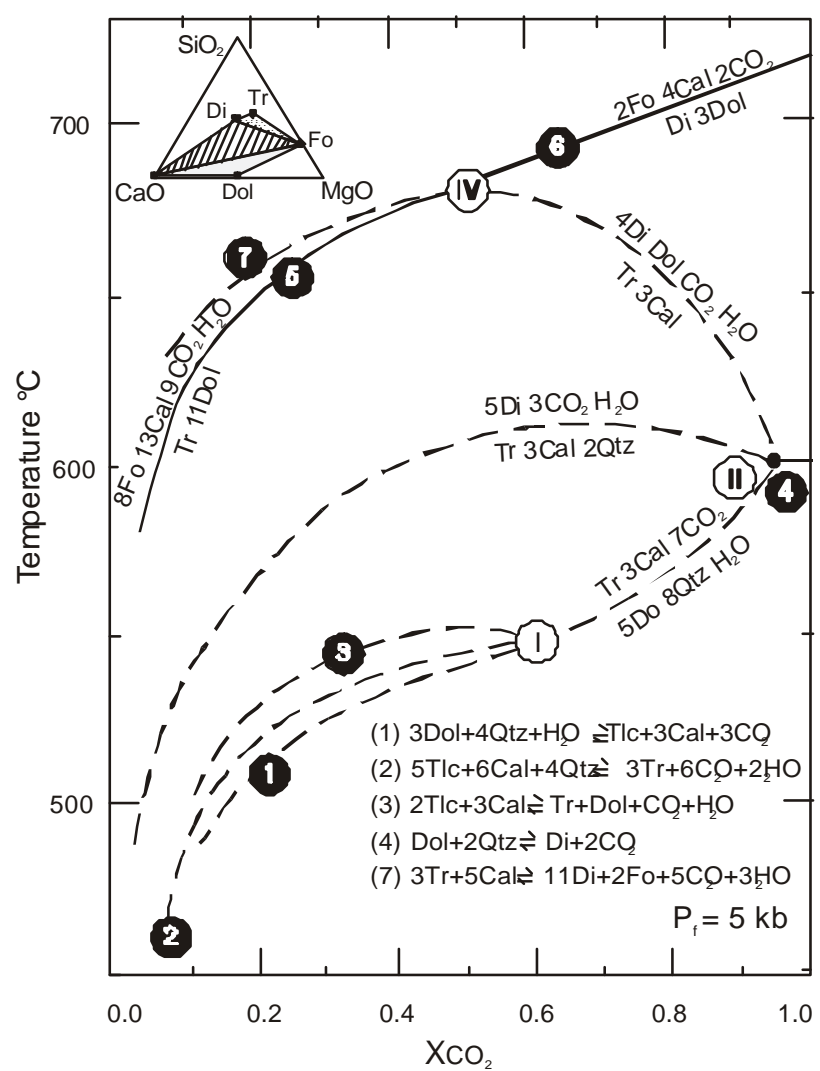

Figure 8: Isobaric T- $\mathrm{CCO}_{2}$ diagram for reactions in siliceous dolomites (Winkler, 1979). 


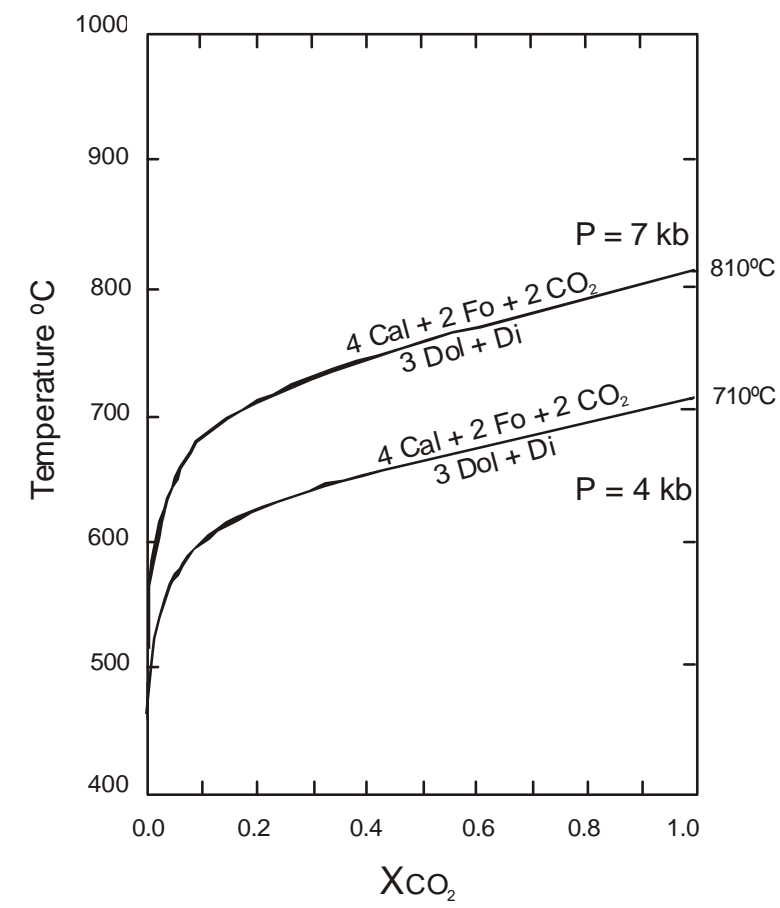

Figure 9: $\mathrm{T}-\mathrm{XCO}_{2}$ diagram showing equilibrium conditions of the reaction $4 \mathrm{Cal}+2 \mathrm{Fo}+2 \mathrm{CO}_{2}=3 \mathrm{Dol}+\mathrm{Di}$ for $\mathrm{P}_{\mathrm{T}}$ values of $4 \mathrm{~kb}$ and $7 \mathrm{~kb}$ in a serpentine marble sample (Berman, 1991).
10) associations will appear, which were not observed. The change of olivine to serpentine must be related to a lowtemperature hydrothermal/metasomatic process.

The aluminous gneiss presents K-feldspar, quartz, plagioclase, cordierite, garnet, sillimanite and gahnitic hercynite, as a high-grade mineral association.

Phase relations concerning these minerals were analysed by various authors (Hensen, 1971, 1986, 1987; Hensen \& Green, 1973; Waters, 1991; Spear, 1993). The phase relations are shown on the diagram of Figure 11, with a Grt-Crd-Sil field in evidence, as well as one for Spl-Qtz-Crd, separated by the reaction Grt+Crd+Sil= Spl+Qtz. It is important to mention, however, that these phase relations are true when spinel contains little or no zinc, whilst the spinel in the analysed samples is fairly rich $(6-11 \%)$ in this element. Granulite facies rocks often exhibit hercinitic spinels with variable $\mathrm{ZnO}$ content, e. g., Adirondacks (Stoddard, 1979); Ardèche (Weber \& Barbey, 1986); Central Australia (Clarke et al., 1990); Eastern Ghats Belt, India (Dasgupta et al., 1995), Mariana - Minas Gerais State, Brazil (Candia, pers. comm.).

The influence of zinc over the spinel stability field has been analysed by Nichols et al. (1992). This element increases the spinel stability field to higher pressures and
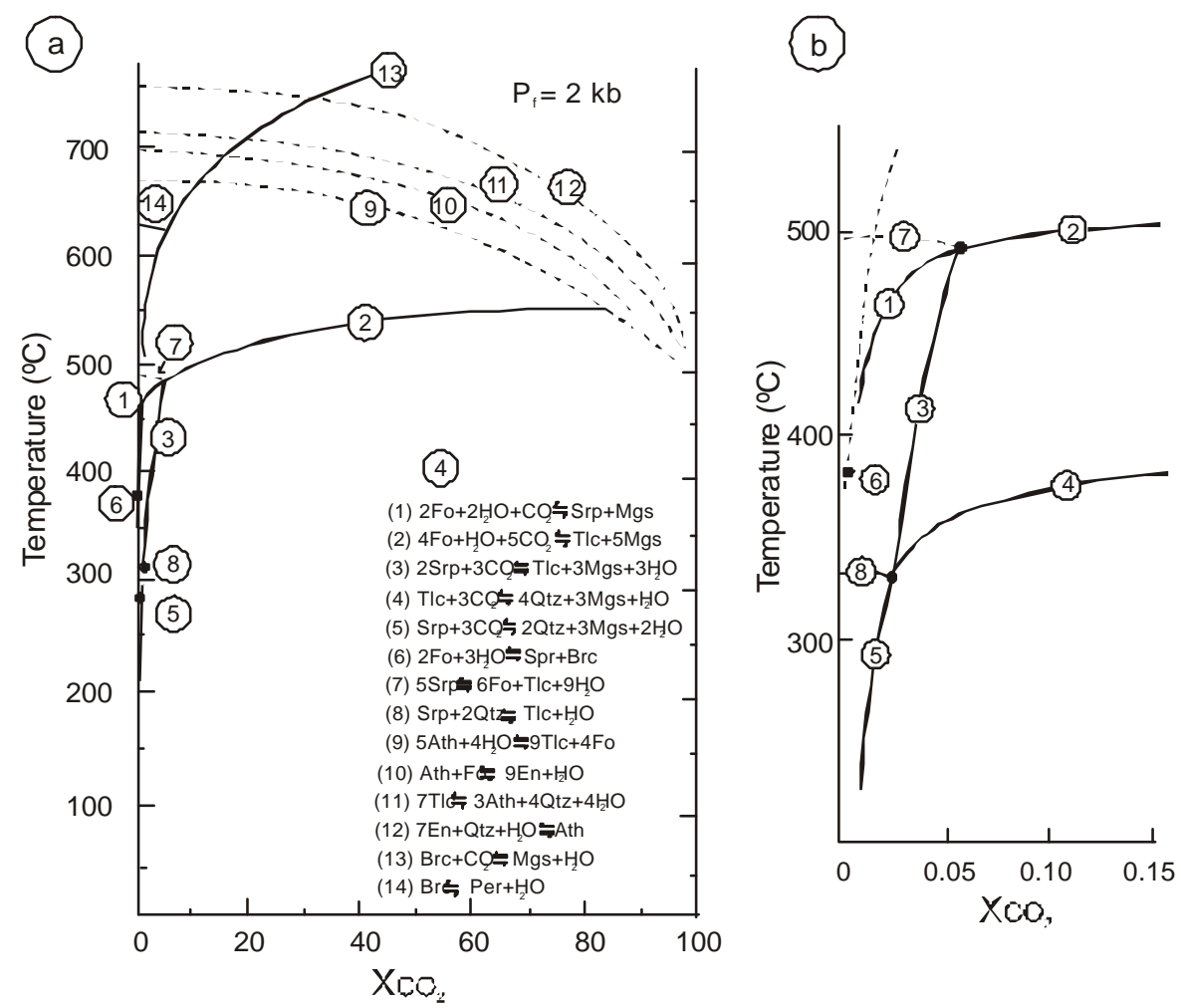

Figure 10: a- Isobaric equilibrium reaction curves in the system $\mathrm{MgO}-\mathrm{SiO}_{2}-\mathrm{H}_{2} \mathrm{O}$ $\mathrm{CO}_{2}$ (Johannes, 1969; simplified). b- Detail of reactions 3 and 5. 


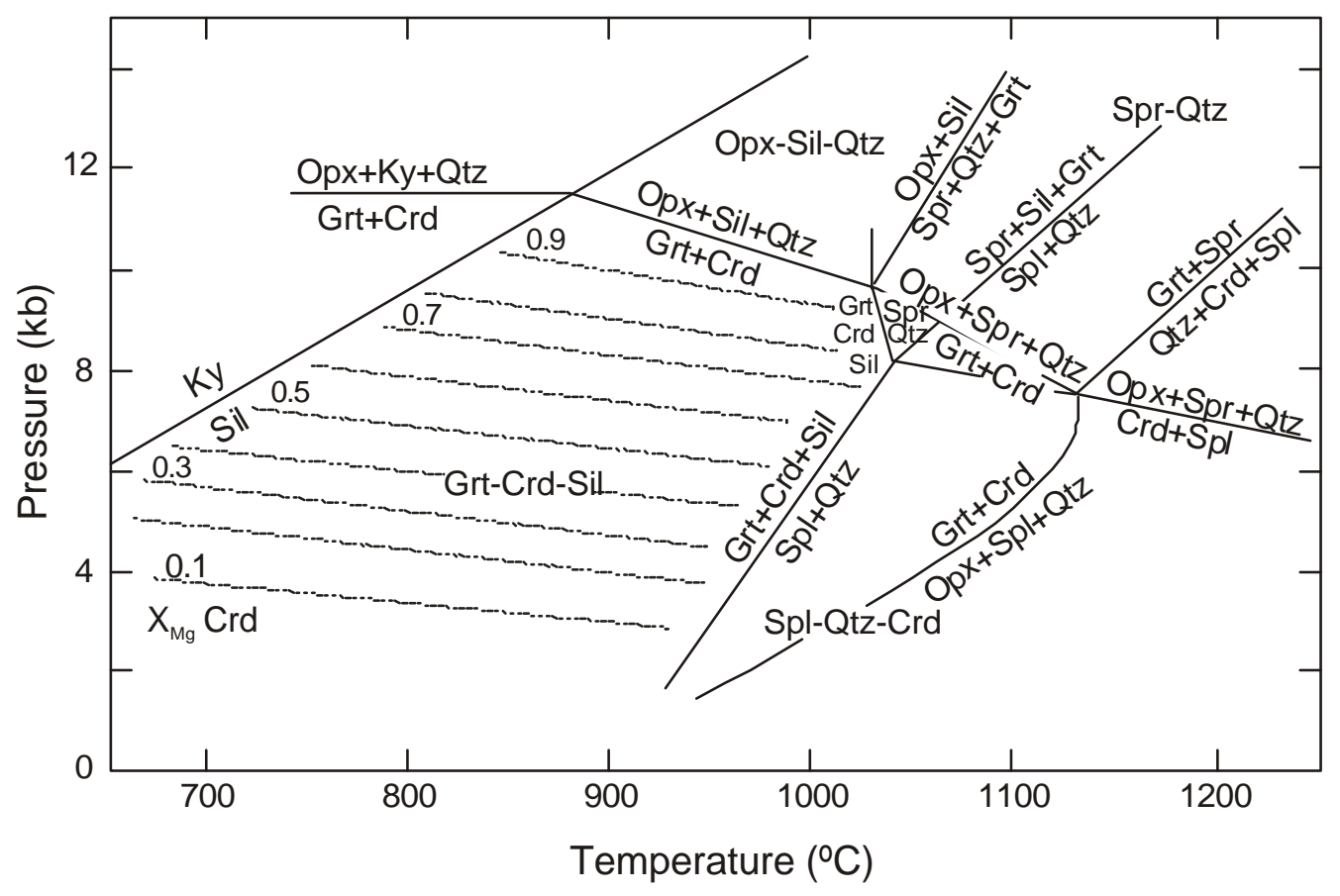

Figure 11: P-T diagram showing Grt-Crd-Sil field at low oxygen fugacity. Isopleths for $X_{\mathrm{Mg}}$ in cordierite (after Hensen $\&$ G reen, 1973).

lower temperatures. Eventually the presence of zinc stabilizes spinel in the Crd-Grt-Sil association.

The stability field for this association is fairly large, attaining $900-1,000^{\circ} \mathrm{C}$, depending on the pressure. To better delimitate the P-T field, Spl-Crd-Sil-Qtz thermobarometry, which takes into account $\mathrm{Zn}$ as a component, was applied (Nichols et al., 1992), thus obtaining $765^{\circ} \mathrm{C}$ and pressures between $8.7 \mathrm{~kb}$ (in water-saturated systems FASHZn and MASHZn) and $6.2 \mathrm{~kb}$ (in dry systems FASZn and MASZn) for spinels included in garnet (Figure $12 \mathrm{a}$ ). The $8.5 \mathrm{~kb}$ pressure is obtained also through the Crd-Spl-Qtz barometer (Perchuk, 1991).

Nichols et al. (1992) also developed a Grt-Crd-Sil-Qtz thermobarometer which yielded P-T conditions of $680^{\circ} \mathrm{C}$ and 6.1-4.6 kb (for garnet cores and more magnesian cordierite) and $530^{\circ} \mathrm{C}$ and $4.5 \mathrm{~kb}$ (for garnet rims and more iron-rich cordierite), which are lower than those obtained through the 2.02 TWQ version (Berman, 1991), respectively of $698^{\circ} \mathrm{C}$ and $7.3 \mathrm{~kb}$ and $665^{\circ} \mathrm{C}$ and $6.4 \mathrm{~kb}$ for the same phases (Figure 12b,c). Results are synthesized in Table 4 and Figure 13.

These results indicate high-grade metamorphic conditions, with pressures in the $7-8 \mathrm{~kb}$ range and temperatures around $800^{\circ} \mathrm{C}$. Some results might reflect $\mathrm{Fe}-\mathrm{Mg}$ reequilibria among the phases under decreasing $\mathrm{P}-\mathrm{T}$ con- ditions. These results suggest a high amphibolite to granulite facies metamorphic environment.

An interesting aspect to be further detailed refers to the behaviour of spinel, which exhibits $X_{\mathrm{Mg}}$ both higher and lower than garnet (Figure 14). A similar behaviour is mentioned by Nichols et al. (1992), with spinel $\mathrm{X}_{\mathrm{Mg}}$ either lower, equal to or higher than that of garnet, with the inflection point representing the appearance of cordierite in the association.

The above mentioned P-T conditions already embrace the anatexis field for rocks of suitable compositions, depending on fluid availability. Anatectic pockets and pegmatoid veins can be locally observed in the outcrops.

\section{METASOMATIC PROCESSES}

The country-rocks, as well as the ultramafic sequence, are strongly affected by metasomatic processes. The common and expressive occurrence of phlogopite or biotite sometimes together with amphibole, indicates a strong secondary transformation of these rocks.

Phlogopite is Ba-rich, element that may be brought by metasomatic fluids. Serpentinization of the olivine "balls" and the phlogopitization of the marbles could be due to 

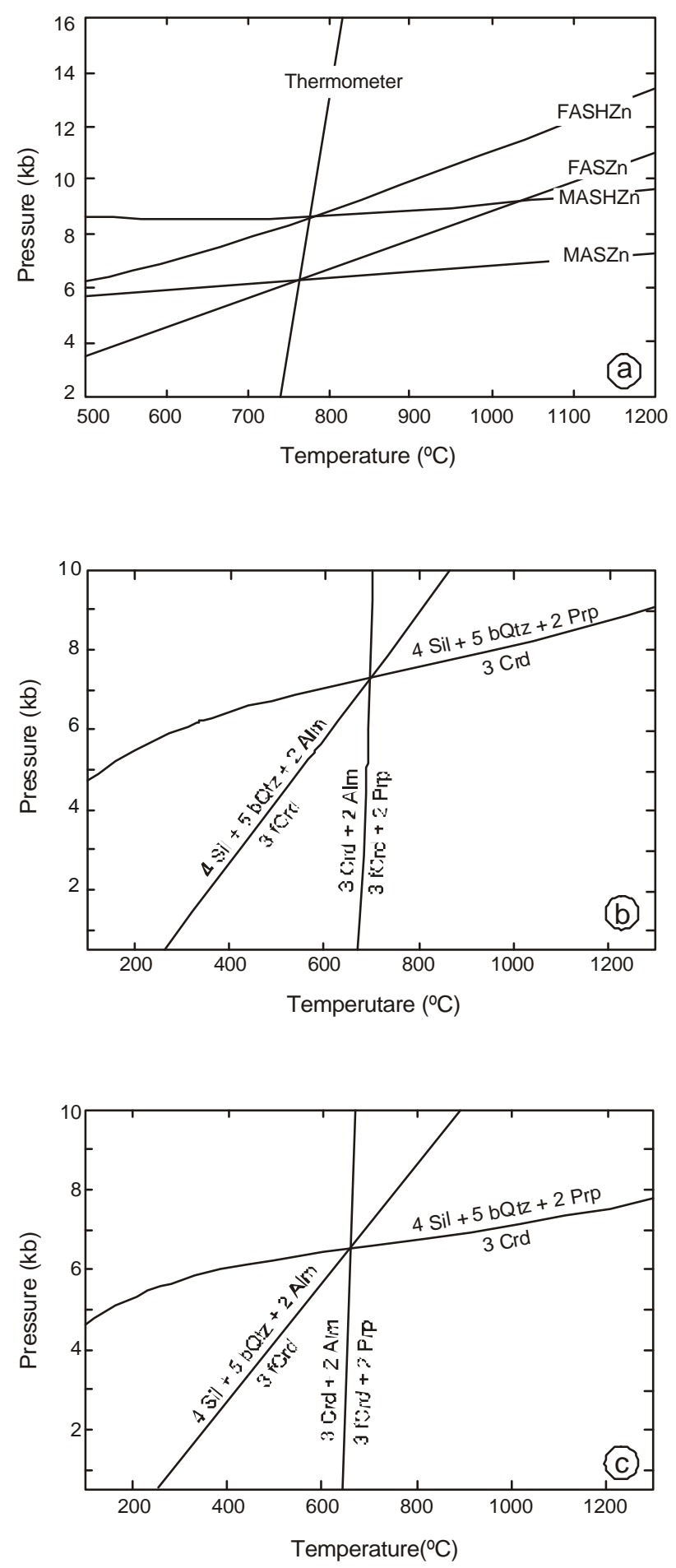

Figura 12: a- P-T diagram for aluminous gneiss showing the Spl-Crd-Sil-Q $女$ thermobarometer of $\mathrm{N}$ ichols et al. (1992). b- P-T diagram for aluminous gneiss showing thermobarometric results by TWQ (Berman, 1991) for garnet core. c- As above, for garnet rim.

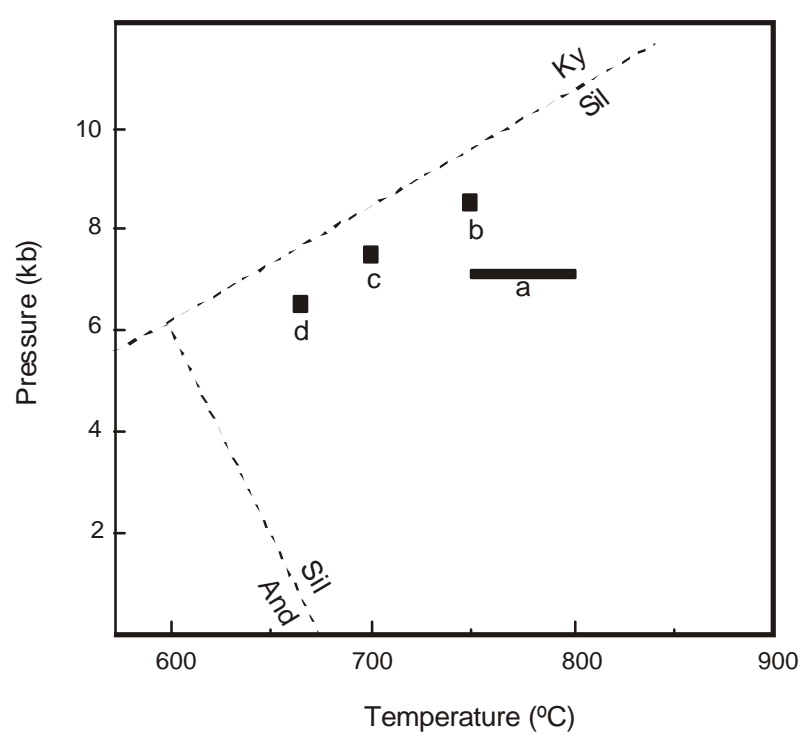

Figure 13: P-T diagram for marble (a) and aluminous gneiss (b, c, d). (b) was obtained by Spl-Crd-Sil-Q $\square$ (Nichols et al., 1992) and Crd-Spl-Q $女$ (Perchuk, 1991) associations. (c) and (d ) were obtained by TWQ (Berman, 1991) for garnet core and rim.

this low-temperature event. Phlogopitization is later than the serpentinization, since phlogopite crystals cut serpentinized minerals.

Besides phlogopite, the presence of apatite (F-rich) and sphene, high-Ba feldspar-bearing rocks and rocks with epidote/clinozoisite and/or garnet are complementary evidences for a metasomatic event.

Kempton (1987) claims that mica introduction into a system increases the amount of some elements, such as Ti, $\mathrm{Al}, \mathrm{Rb}, \mathrm{Sr}$ and $\mathrm{Ba}$. Following this, $\mathrm{Ba}$ found in some $\mathrm{K}-$ feldspar and $\mathrm{Sr}$ in apatite could have been originated from the phlogopitization process or derived from late hydrothermal infiltration.

\section{CONCLUSIONS}

The chromite bearing mafic-ultramafic rocks of the Jacurici Valley are deformed, metamorphosed and transformed by metasomatic processes, making it difficult to interpret their evolutive record.

In spite of the metasedimentary country-rocks having also been affected by the processes mentioned above, it is possible to recover their metamorphic record. These rocks were studied to aid in the interpretation of the metamorphic/evo- 
Table 4: Thermobarometric data for aluminous gneiss.

\begin{tabular}{cccrr}
\hline & TWQ & SPL-CRD-SIL-QTZ & GRT-CRD-SIL-QTZ & CRD-SPL-QTZ \\
& GRT-CRD-SIL & (Nichols et al., 1992) & (Nichols et al., 1992) & (Perchuck, 1991) \\
Grt core & $698^{\circ} \mathrm{C} \mathrm{e} \mathrm{7.3} \mathrm{kb}$ & $770^{\circ} \mathrm{C}$ and $8.7 \mathrm{~kb}$ (FMASHZn) & $680^{\circ} \mathrm{C}$ and $6.1 \mathrm{~kb}$ & $8.5 \mathrm{~kb}$ \\
& & $760^{\circ} \mathrm{C}$ and $6.2 \mathrm{~kb}$ (FMASHZn) & $4.6 \mathrm{~kb}$ & \\
Grt rim & $665^{\circ} \mathrm{C} \mathrm{e} 6.4 \mathrm{~kb}$ & & $530^{\circ} \mathrm{C}$ and $4.5 \mathrm{~kb}$ & \\
\hline
\end{tabular}
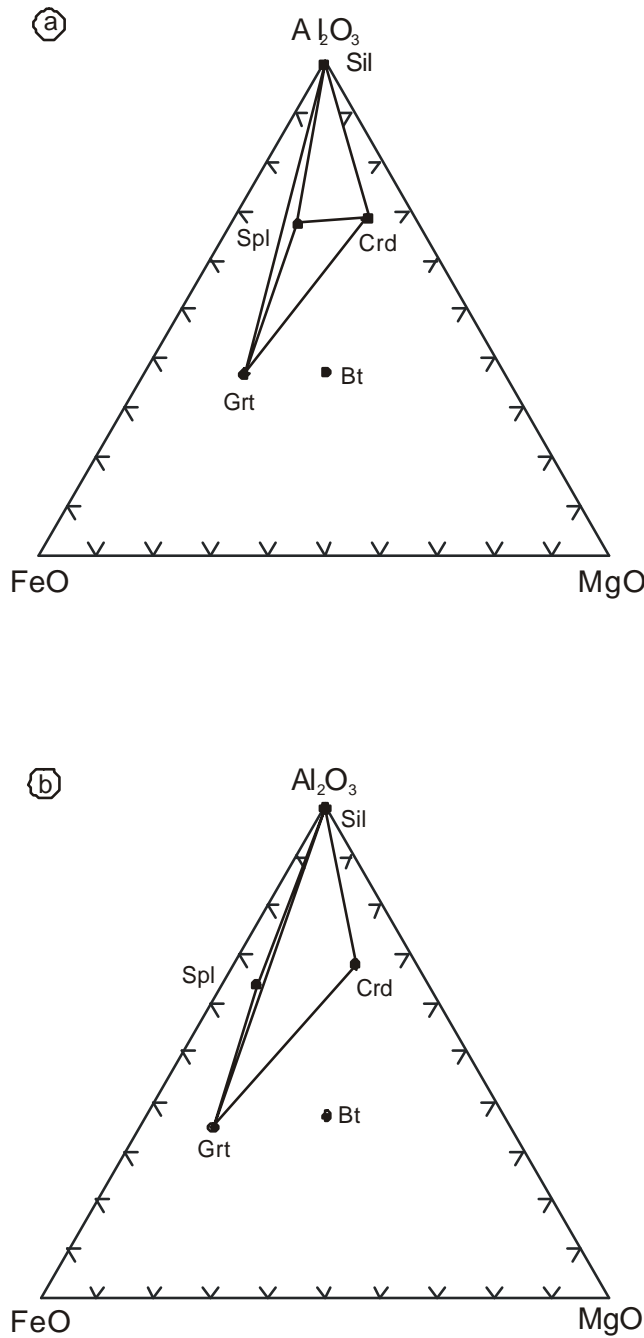

Figure 14: AFM compatibility relations for aluminous gneiss. a- For garnet core and $\mathrm{Mg}$-richer spinel $\left(X_{\mathrm{Mg}}^{\text {spinel }}>X_{\mathrm{Mg}}^{\text {garnet }}\right)$, b- For garnet rim and $\mathrm{Mg}$-poorer spinel $\left(X_{M g}{ }^{\text {spinel }}<X_{M g}{ }^{\text {garnet }}\right)$. lutionary history of the area. In the pelitic metasediments the data obtained indicate P-T conditions for the highest metamorphic record around $750-800^{\circ} \mathrm{C}$ and $7-8 \mathrm{~kb}$.

The rocks of this sequence were subjected to strong phlogopitization. It is not clear whether there was only one metasomatic event or various metasomatic episodes. Phlogopitization is observed to have happened after serpentinization, and also after the carbonatation episode, but it has not been possible so far to establish the chronological relationship of this last event.

\section{ACKN O WLEDGEMENTS}

The authors thank FAPESP for the pos-doctoral fellowship (proc. $\left.n^{\circ} .98 / 00681-6\right)$ to Del Lama and Research Project financing (proc. $n^{\circ} .97 / 00640-5$ ); and Prof. Dr. Antenor Zanardo for the valuable petrographic discussions.

\section{REFERENCES}

BARBOSA, J.S.F.; DOMINGUEZ, J.M.L. (1996) Geologia da Bahia: texto explicativo para o mapa geológico ao milionésimo. Salvador, SGM. 400 p.

BARBOSA, O. (1964) Geologia de parte da região do médio São Francisco, Nordeste do Brasil. Petropólis, Prospec. 69 p.

BARBOSA, O. (1970) Geologia de parte da região do médio São Francisco, Nordeste do Brasil. Boletim DNPM/DFPM, n. 140, p. 1-98.

BARBOSA DE DEUS, P.; VIANA, J.S. (1982) Jacurici valley chromite district. In: INTERNATIONAL. SYMPOSIUM ON ARCHEAN AND EARLY PROTEROZOIC GEOLOGIC EVOLUTION AND METALLOGENESIS, Salvador, 1982. Abstracts and Excursions. Salvador, SBG/CPM. p. 97-107.

BERMAN, R.G. (1991) Thermobarometry using multiequilibrium calculations: a new technique, with petrological applications. Canadian Mineralogist, v. 29, p. 833-855. 
CLARKE, G.L.; COLLINS, W.J.; VERNON, R.H. (1990) Sucessive overprinting granulite facies metamorphic events in the Anmatjira Range, central Australia. Journal of Metamorphic Geology, v. 8, n. 1, p. 65-88.

DASGUPTA, S.; SENGUPTA, P.; EHL, J.; RAITH, M.; BARDHAN, S. (1995) Reaction textures in a suite of spinel granulites from the Eastern Ghats Belt, India: evidence for polymetamorphism, a partial petrogenetic grid in the system KFMASH and the roles of $\mathrm{ZnO}$ and $\mathrm{Fe} 2 \mathrm{O} 3$. Journal of Petrology, v. 36, n. 2, p. 435-461.

FIGUEIREDO, M.C.H. (1981) Geoquímica das rochas metamórficas de alto grau no nordeste da Bahia, Brasil. In: INCA, H.A.V. Geologia e recursos minerais do Estado da Bahia: textos básicos. Salvador, SGM/CPM. v. 4, p. 1-71.

HENSEN, B.J. (1971) Theoretical phase relations involving cordierite and garnet in the system $\mathrm{MgO}-\mathrm{FeO}$ $\mathrm{A} 12 \mathrm{O} 3-\mathrm{SiO} 2$. Contributions to Mineralogy and Petrology, v. 33, n. 3, p. 191-214.

HENSEN, B.J. (1986) Theoretical phase relations involving cordierite and garnet revisited: the influence of oxygene fugacity on the stability of sapphirine and spinel in the system Mg-Fe-Al-Si-O. Contributions to Mineralogy and Petrology, v. 92, n. 3, p. 362-367.

HENSEN, B.J. (1987) P-T grids for silica-undersatured granulites in the systems MAS $(n+4)$ and FMAS (n +3 ) - tools for the derivation of P-T paths of metamorphism. Journal of Metamorphic Geology, v. 5, n. 2, p. 255-271.

HENSEN, B.J.; GREEN, D.H. (1973) Experimental study of the stability of cordierite and garnet in pelitic compositions at high pressures and temperatures III. Synthesis of experimental data and geological applications. Contributions to Mineralogy and Petrology, v. 38, n. 2, p. 151-166.

JARDIM DE SÁ, E.F. (1984) Geologia da região do Vale do Jacurici. Natal, 17p. (Relatório de Consultoria para CPM).

JOHANNES, W. (1969) An experimental investigation of the system MgO-SiO2-H2O-CO2. American Journal of Science, v. 267, n. 9, p. 1083-1104.

KEMPTON, P.D. (1987) Mineralogic and geochemical evidence for differing styles of metasomatism in spinel lherzolite xenoliths: enriched mantle source regions of basalts? In: MENZIES, M.A.; HAWKESWORTH, C.J. (eds.) Mantle metasomatism London, Academic Press, p. 45-89. (Academic Press Geology Series).
KRETZ, R. (1983) Symbols for rock-forming minerals. American Mineralogist, v. 68, n. 1/2, p. 277-279.

NICHOLS, G.T.; BERRY, R.F.; GREEN, D.H. (1992) Internally consistent gahnitic spinel-cordierite-garnet equilibria in the FMASHZn system: geothermobarometry and applications. Contributions to Mineralogy and Petrology, v. 111, n. 3, p. 362-377.

OLIVEIRA, E.P. (1998) The Cu-rich Caraíba and Cr-rich Medrado mafic-ultramafic complexes, Bahia, Brazil: $\mathrm{U}-\mathrm{Pb}$ and $\mathrm{Nd}$ isotope constraints for archaean lithospheric mantle remobilisation in the Paleoproterozoic Salvador-Curaça Orogen. In: CONGRESSO BRASILEIRO DE GEOLOGIA, 40, Belo Horizonte, 1998. Anais. Belo Horizonte, SBG. p. 48.

PERCHUK, L.L. (1991) Derivation of a thermodynamically consistent set of geothermometers and geobarometers for metamorphic and magmatic rocks. In: PERCHUK, L.L. (ed.) Progress in metamorphic and magmatic petrology. A memorial volume in honor of D. S. Korzhinskiy. Cambridge, University Press. p. 93-112.

SILVA, L.C.; McNAUGHTON, N.J.; MELO, R.C.; FLETCHER, I.R. (1997) U-Pb SHRIMP ages in the Itabuna-Caraíba TTG high-grade complex: the first window beyond the Paleoproterozoic overprinting of the eastern Jequié craton, NE Brazil. In: INTERNATIONAL SYMPOSIUM ON GRANITES AND ASSOCIATED MINERALIZATIONS, 2, Salvador, 1997. Extended Abstract and Program. Salvador, SGM. p. 282-283.

SPEAR, F.S. (1993) Metamorphic phase equilibria and pressure-temperature-time paths. Washington, Mineralogical Society of America. 799p. (Monograph).

STODDARD, E.F. (1979) Zinc-rich hercynite in highgrade metamorphic rocks: a product of the dehydration of staurolite. American Mineralogist, v. 64, n. 7/8, p. 736-741.

WATERS, D.J. (1991) Hercynite-Quartz granulites: phase relations and implications for crustal processes. European Journal of Mineralalogy, v. 3, n. 2, p. 367-386.

WEBER, C.; BARBEY, P. (1986) The role of water, mixing process and metamorphic fabric in the genesis of the Beaume migmatites (Ardèche, France). Contributions to Mineralogy and Petrology, v. 92, n. 4, p. 481-491.

WINKLER, H.G.F. (1979) Petrogenesis of metamorphic rocks. New York, Springer-Verlag, 348p. 